\title{
MEDIOAMBIENTE O SUPERVIVENCIA: LOS DESAFIOOS Y OPORTUNIDADES DE LAS MICROEMPRESAS EN LA ECONOMÍA CIRCULAR
}

\author{
ENVIRONMENT OR SURVIVAL: THE CHALLENGES \\ AND OPPORTUNITIES OF MICRO-ENTERPRISES \\ IN THE CIRCULAR ECONOMY
}

\author{
Eric Alfonso Castro Góngoral, Jennifer Mul Encalada²
}

\begin{abstract}
Resumen
En la actualidad se ha generado un nuevo modelo de economía denominado economía circular cuyo objetivo es reducir la producción y el consumo de materiales y energía por medio de ciclos renovables. Este modelo puede ser aplicado al interior de las microempresas, sin embargo, su implementación genera desafios que muchas veces no pueden ser superados con facilidad, lo que ocasiona que los microempresarios tengan que decidir entre aplicar un modelo que apoya al medio ambiente o perpetuar el modelo lineal (más accesible) con el fin de sobrevivir en el mercado. Por lo tanto, el objetivo del presente trabajo es analizar los desafíos y las oportunidades de la implementación de la economía circular en las microempresas. Para ello se llevó a cabo una revisión documental con el fin de validar el fenómeno que se estudia. En los resultados se observa que la economía circular ha generado importantes beneficios económicos en Europa, pero es desaprovechada en los países subdesarrollados debido principalmente a los problemas estructurales de la región. Se concluye que la economía circular proporciona grandes oportunidades a las microempresas, pero también representa grandes retos que los negocios tienen que enfrentar para su implementación.
\end{abstract}

\section{Palabras clave}

Economía circular, desafios, oportunidades, microempresa, medio ambiente.

\begin{abstract}
At present, a new economic model called the circular economy has been generated whose objective is to reduce the production and consumption of materials and energy through renewable cycles. This model can be applied within microenterprises, however, its implementation generates challenges that often cannot be easily overcome, which causes microentrepreneurs to decide between applying a model that supports the environment or perpetuating the model linear (more accessible) to survive in the market. Therefore, the objective of this work is to analyze the challenges and opportunities of the implementation of the circular economy in micro-enterprises. For this, a documentary review was carried out to validate the phenomenon under study. The results show that the circular economy has generated important economic benefits in Europe but is wasted in underdeveloped countries mainly due to structural problems in the region. It is concluded that the circular economy provides great opportunities for micro-enterprises but also represents great challenges that businesses have to face for its implementation.
\end{abstract}

\section{Keywords}

Circular economy, challenges, opportunities, micro-business, environment.

JEL: M21 


\section{Introducción}

Desde el inicio de la primera Revolución Industrial se ha seguido un modelo lineal de producción y consumo en el que se generan productos que se venden, se utilizan y se desechan como residuos. Este tipo de modelo ha ocasionado que los recursos del planeta se hayan sobre explotado y que en la actualidad existan problemas como la contaminación del agua en comunidades, la deforestación sin control y bosques y selvas convertidos en basureros a cielo abierto. Las grandes fábricas han ocasionado un incremento en las cantidades de dióxido de carbono en la atmósfera, los efectos del cambio climático se han vuelto más evidentes y la contaminación de océanos y mares con "islas" de plásticos es más que evidente. Para el año 2030 se calcula un aumento de tres billones de consumidores que generarán una importante demanda de energía. Es esencial revertir la lógica de descartar los desperdicios por un modelo donde estos se reutilizan y valorizan (Lett, 2014). Para el año 2050 habrá aproximadamente 9 billones de personas viviendo en el planeta. Lo anterior llevará a que la economía global requiera un 300 \% más de los recursos que se utilizan actualmente. En la economía lineal aproximadamente el $80 \%$ de lo que utilizamos es descartado después de su uso. El 99 \% del material total generado para producir bienes para el consumo termina en un depósito de desechos en un período de seis meses (Planing, 2015). Ante esta situación, ha surgido un nuevo modelo de economía circular que tiene como objetivo la generación de prosperidad económica mediante la protección del medio ambiente y la prevención de la contaminación para de esta forma facilitar el desarrollo sostenible. La preocupación por el uso adecuado de los recursos naturales y la calidad del medio ambiente no es un tema nuevo para la sociedad actual, ya que, esta situación ha sido discutida por ambientalistas desde los años 60 del siglo XX (Prieto, Jaca y Ormazabal, 2017). Surge como necesidad apremiante un nuevo concepto de desarrollo, el 'desarrollo sostenible' definido como aquel que garantiza las necesidades del presente sin comprometer las posibilidades de las generaciones futuras para satisfacer sus propias necesidades (Organización de las Naciones Unidas, 1987).

En ese escenario surge el modelo de la economía circular como una opción ante el modelo de economía lineal. La economía circular permite una mejor reacción ante los desafíos de la producción basada en la extracción, transformación y distribución indiscriminada de materias primas y energía en productos y servicios para los mercados. El modelo de economía circular se basa en un sistema de aprovechamiento de recursos en donde la reducción, la reutilización y el reciclaje son elementos fundamentales para el crecimiento económico y el cuidado del medio ambiente. A nivel global, la economía circular puede ayudar a las naciones en desarrollo a industrializarse y a los países desarrollados a incrementar el bienestar y reducir la variación en el precio de los recursos sin colocar presión sobre los recursos naturales o los limites ambientales (Preston, 2012).

La incorporación del modelo de economía circular en la sociedad, el gobierno y el sector empresarial se ha convertido en una tarea fundamental en los años recientes, sin embargo, es a nivel empresarial, específicamente en el marco de la micro y pequeña empresa que esta noción toma mayor importancia debido a las ventajas y los beneficios que la aplicación del modelo conlleva para su desarrollo y por la trascendencia que tienen este tipo de organizaciones en la disminución de la pobreza y el desempleo en los países en vías de desarrollo. De acuerdo con Veolia México (2018), la economía circular no solo contribuye a la preservación del medio ambiente, sino que también le brinda una 
oportunidad a las empresas para reducir sus costos y crear modelos económicos nuevos y virtuosos a largo plazo.

A pesar de las ventajas que la implementación de la economía circular aporta a las microempresas, también existen riesgos que tienen que contrarrestarse para que las empresas puedan aprovechar dichas ventajas. Entre los principales riesgos se encuentran: la dependencia de la industria a los combustibles fósiles, el interés de los grupos en el poder, el incremento en los costos de inversión de los negocios en el corto plazo y el bajo valor que los consumidores pueden otorgar a los productos derivados de la economía circular, entre otros (Preston, 2012). Desgraciadamente en la lucha diaria de las microempresas por sobrevivir y generar utilidades, el modelo lineal sigue siendo muy relevante debido a la falta de acceso al capital necesario para la implementación de la economía circular en las microempresas. Los problemas económicos de los países subdesarrollados han propiciado que los microempresarios se enfoquen en visiones de corto plazo y no puedan explorar otras alternativas más amigables con el planeta.

\section{Marco teórico}

\section{El modelo de economía lineal}

De acuerdo con la fundación Ellen MacArthur (2017) la economía a nivel mundial se ha encontrado desde los inicios del capitalismo dominada por un modelo de economía lineal de producción y consumo, a través del cual se generan productos a partir de una gran cantidad de materias primas que luego se venden, se utilizan y, posteriormente, se desechan como residuos. Aunque con el paso del tiempo se han logrado importantes avances en materia de la eficiencia en el uso de los recursos al momento de realizar un proceso productivo, dichos avances, resultan insuficientes si se basan exclusivamente en el consumo de materiales. A partir del siglo XX, la rápida expansión de las economías en el mundo ha provocado un incremento de aspectos negativos para el medio ambiente y es muy probable que la tendencia continúe para los próximos años. Trabajar hacia la eficiencia como solución (una reducción de los recursos y disminución de la energía no renovable utilizada en la producción) no cambia la naturaleza finita de los recursos, sino que solamente se intenta retrasar lo inevitable:

1. El modelo actual genera una gran cantidad de residuos de forma diaria. En Europa solo se recicla el $5 \%$ del total de los recursos.

2. Muchos países cuentan con pocos depósitos de recursos naturales no renovables por lo que tienen que depender al $100 \%$ de las importaciones.

3. El modelo lineal ha generado una gran cantidad de problemas medioambientales que se agravarán en el futuro. Se han agotado una cantidad increíble de reservas, se está generando un cambio climático impactante, se pierde una gran parte de la biodiversidad y se contaminan la tierra y los océanos.

4. En los últimos años las empresas se han enfrentado a cambios en las regulaciones que intentan aminorar los efectos negativos del modelo lineal.

\section{El modelo de economía circular}

La economía circular es un nuevo modelo económico que promueve que los recursos se empleen de una manera más sostenible y eficiente dando lugar a una economía más competitiva y baja en carbono. Se consideran los ciclos de vida de los productos, desde el diseño o la producción a la gestión de residuos. La economía circular no solo tiene beneficios económicos sino también ambientales. Contribuye a la conservación del capital natural 
a través de reducir o evitar daños a la biodiversidad, la contaminación del aire, el suelo o el agua, poniendo foco también en la lucha contra el cambio climático (Comisión Europea para la Economía Circular, 2015). La economía circular es vista como un importante mecanismo para la promoción de la producción sustentable o como un paradigma futura que cambiará la industria de la transformación (Korhonen, Nuur, Feldmann, Eshetu, 2018). Para continuar con esta idea, García y Ortega (2018) comentan que la economía circular se plantea como una medida más revolucionaria que lleve a las empresas al ahorro de recursos, al mismo tiempo que al consumidor a un cambio en sus hábitos de compra, ambos con un único objetivo en común; el cual es el cuidado del medioambiente. Pero si bien es cierto, este cambio no se logrará de la noche a la mañana, requiere de la colaboración dinámica de los agentes sociales (sociedad, empresas, gobierno) para poder llevarla a cabo de manera sustentable y certera.

De acuerdo con Prieto, Jaca y Ormazabal (2017), la economía circular consiste en un flujo cíclico, que implica extraer, transformar, distribuir, usar y recuperar los materiales y la energía de productos y servicios. La economía circular cuenta con cinco campos de acción, los cuales se mencionan a continuación:

1. Extraer: el término extraer se refiere a la forma en que las industrias toman recursos del entorno, por tanto, las empresas deben intentar hacer un uso más eficaz y responsable de los recursos biológicos y técnicos. Esto implica que las empresas pueden seleccionar los proveedores y los materiales que utilizan, de acuerdo con criterios medioambientales que disminuyan su impacto en la naturaleza.

2. Transformar: tan pronto se obtienen los recursos, se debe procurar el desarrollo de las mejores prácticas tecnológicas e innovaciones ecológicas (eco-innovaciones) para que tanto el producto o servicio como su proceso se realicen de la manera más sostenible posible.

3. Distribuir: esta fase tiene que ver con la forma en la que el producto o servicio se entrega al cliente. Las empresas deben garantizar la trazabilidad de sus productos y de manera eficiente reducir el impacto ambiental, tanto en rutas, como embalajes, como, a través de distintas prácticas, como la logística inversa.

4. Usar: en cuanto el producto está a disposición de los consumidores u otras empresas, la economía circular propone reducir el impacto de la energía asociada al uso del producto o la eficiencia del propio producto. La eficiencia del producto o servicio puede ser mejorada a través de la reutilización como producto de segunda mano o la reparación.

5. Recuperar: por último, en la economía circular, los residuos pueden ser recuperados de dos maneras: como un recurso biológico que puede ser devuelto a la biósfera o como un recurso técnico que puede ser reincorporado a un proceso industrial.

\section{Ventajas generadas por la economía circular}

Los campos de acción que se describen pueden generar ventajas para diversos sectores. Los principales sectores que son beneficiados con el empleo del modelo de la economía circular son la economía en general, el medioambiente y las empresas. De acuerdo con Espaliat (2017), existen tres diferentes tipos de ventajas que se generan al emplear la economía circular: ventajas económicas, ventajas ambientales y ventajas empresariales. A continuación se habla de las ventajas mencionadas: 
Ventajas económicas de la estrategia circular

- Crecimiento económico

- Ahorros netos de costes de materias primas

- Creación de empleo

Ventajas ambientales de la estrategia circular

- Prevención de riesgos y gestión equilibrada de los recursos naturales

- Reducción de emisiones de Dióxido de Carbono $\left(\mathrm{CO}_{2}\right)$

- Reducción del consumo de materias primas

Ventajas empresariales de la estrategia circular

- Incremento de la productividad y la competitividad: reduce costos y dependencia de recursos primarios.

- Generación de beneficios: genera flujos de ventas y utilidades nuevos.

- Reducción de la volatilidad de los precios e incremento de la seguridad de los suministros: mayor uso de insumos reciclados.

- Generación de demanda de nuevos servicios empresariales: como ejemplos tenemos la logística inversa, nuevas formas de comercialización y la creación de nuevas piezas o componentes.

- Aumento de la calidad y reducción del precio de productos y servicios: se satisfacen mejor las necesidades de los clientes.

- Reducción de la obsolescencia: incremento en el ciclo de vida útil de los productos.

Por otro lado, de acuerdo a la Comisión Europea para la Economía Circular (2015) existen cinco beneficios empresariales de adoptar prácticas de economía circular en las microempresas. Los beneficios se mencionan a continuación:
- Menor exposición al aumento y la volatilidad de los precios de los recursos: los recursos naturales no renovables (por ejemplo, combustibles fósiles, metales y minerales) son cada vez más escasos.

- El pensamiento circular estimula la innovación en las empresas: el concepto de economía circular proporciona inspiración para que las empresas aumenten la eficiencia de sus recursos.

- Crea una imagen verde: los consumidores, las empresas y los gobiernos son cada vez más conscientes del impacto ambiental de los productos que utilizan.

- Abre nuevos mercados y oportunidades para crecer: las soluciones circulares también pueden crear nuevos mercados o nichos.

- Aumento de la lealtad del cliente y flujo de ingresos más estable: Volverse más circular puede facilitarse adoptando un modelo comercial diferente.

\section{Estrategias de implementación de la economía circular}

De acuerdo con Kalmykova, Sadagopan, Rosado (2018) y con Ludeke, Gold, Bocken (2018) existen una gran variedad de estrategias a través de las cuales es posible implementar la economía circular en las microempresas. Algunas de las más importantes se presentan a continuación:

- Diseñar productos de una mayor duración.

- Diseñar métodos para extender la vida de los productos.

- Diseñar métodos de reciclaje.

- Diseñar las formas de re usar los productos.

- Producción autónoma de energía.

- Búsqueda de proveedores eco amigables. 
- Planeación de la producción ecológica de los productos.

\section{Barreras para la implementación de la economía circular en microempresas}

A pesar de la implementación generalizada de la economía circular, este enfoque requiere de cambios profundos en la industria y patrones de consumo. Las industrias y la producción concentran algunas de las mayores barreras para implementar la economía circular en las microempresas. Las principales barreras se exponen a continuación:

- Bloqueo de infraestructura en recursos y modelos de desarrollo: la producción, el consumo y el comercio son altamente dependientes de combustibles fósiles y orientados a la fabricación de un solo uso.

- Obstáculos políticos: regulaciones con profundos impactos sistémicos pueden ser frustrados y debilitados por un interés especial de algunos grupos.

- Altos costos iniciales: a nivel macro, una economía circular exitosa fomentaría el crecimiento y reducir la vulnerabilidad a los choques de precios de los recursos. Pero a corto plazo, inevitablemente habrá un importante aumento inicial en los costos de inversión y riesgos para las empresas.

- Intentar transformar el modelo de negocio central de una empresa: es una tarea arriesgada en sí misma y se requerirá de un negocio sólido con reglas claras.

- Falta de entusiasmo del consumidor: para que las empresas se beneficien de la producción verde, los consumidores necesitarán comprender y valorar lo que representa el concepto.

- Desafíos para la cooperación empresa a empresa: la incorporación de prácticas de economía circular puede re- querir que múltiples empresas tengan que ajustar sus operaciones.

- El reto de la innovación: para optimizar la cadena de suministro se requerirá de infraestructura y tecnología inteligente (Preston, 2012).

De acuerdo con Garcés, Rivera, Suárez y Leyva (2019), existen dos tipos de barreras o retos para la implementación de la economía circular en microempresas: las barreras duras (falta de apoyo financiero, insuficientes sistemas de gestión de la información, falta de tecnología adecuada, recursos técnicos insuficientes, recursos financieros insuficientes y falta de apoyo por parte de las instituciones públicas y de organizaciones humanas) y las barreras de base humana (falta de interés del cliente en el medioambiente, falta de personal calificado en asuntos ambientales y falta de compromiso de los líderes de la organización).

\section{El papel de la microempresa}

Para observar de forma más clara el impacto que las microempresas tienen en las economías mundiales, Durán (2017) comenta que este tipo de negocios suponen el $90 \%$ del sector empresarial y contribuyen hasta en un $45 \%$ al empleo formal y en un $33 \%$ al ingreso nacional (PIB) en las economías emergentes. De acuerdo a la Secretaría de Economía de México (2010), las microempresas son negocios con menos de diez trabajadores, que generan anualmente ventas hasta por 4 millones de pesos y representan el $95 \%$ del total de las empresas y el $40 \%$ del empleo en el país. El Programa Nacional de Financiamiento al Microempresario en México (2002) menciona que en todo el mundo, la microempresa ha demostrado su capacidad como un medio efectivo para propiciar el desarrollo social.

El papel que las microempresas desempeñan en el mundo contemporáneo es 
convertirse en un factor destacado de resistencia de la población de un país frente a la creciente agudización de los problemas de la economía en general. En los países latinoamericanos, las microempresas se convierten en unidades de producción de bienes y servicios que se añaden de forma importante a la dinámica económica para la satisfacción de las necesidades básicas de la población aunque la mayoría de las veces, dicho proceso no toma en cuenta el cuidado de los recursos ni el medioambiente. Es por lo tanto, muy importante que el gobierno implemente medidas que faciliten la transición de modelos lineales a modelos circulares en las microempresas. Los beneficios económicos y sociales de los negocios, también deberían permear en el mejoramiento del medioambiente.

En casi todos los países del mundo las microempresas comparten ciertos rasgos y características que le proporcionan su carácter único dentro de todos los agentes de la economía. De acuerdo a Sánchez (2007), los rasgos más importantes son: operan con escalas bajas de producción, utilizan tecnologías adaptadas, los empleados no poseen capacitación, se constituyen con poca inversión, no cuentan con visión a largo plazo, carecen de proveedores formales, no acceden a información vital del mercado, son a menudo de propiedad familiar y su financiamiento procede de fuentes propias. Es en este punto, en donde se observan los principales desafíos para la implementación de la economía circular en las microempresas: baja producción, altos costos de implementación, falta de inversión y visión a corto plazo, entre otras.

\section{Metodología}

El término metodología hace referencia al modo en que enfocamos los problemas y buscamos las respuestas, a la manera de realizar la investigación. Nuestros supuestos teóricos y perspectivas, y nuestros propósitos, nos llevan a seleccionar una u otra metodología (Quecedo y Castaño, 2002). Los principales métodos de investigación científica a través de los que se puede estudiar la realidad de un fenómeno son el cuantitativo y el cualitativo. La distinción fundamental entre investigación cuantitativa e investigación cualitativa estriba en el tipo de conocimiento que se pretende. Aunque parezca extraño, la distinción no está relacionada directamente con la diferencia entre datos cuantitativos y datos cualitativos, sino con una diferencia entre búsqueda de causas frente a búsqueda de acontecimientos. Los investigadores cuantitativos destacan la explicación y el control; los investigadores cualitativos destacan la comprensión de las complejas relaciones entre todo lo que existe (Stake, 1998). Mientras que el método cualitativo busco la comprensión de fenómenos de forma no generalizable y con cierta subjetividad, el método cuantitativo se basa en la medición controlada y objetiva de variables para realizar generalizaciones.

Después de analizar los diferentes enfoques y las técnicas de investigación se decide utilizar el enfoque cualitativo a través de la técnica de revisión documental, ya que se efectuaron diversos análisis a través de la búsqueda de literatura especializada para entender mejor un fenómeno. Corbetta (2007) comenta que la sociología puede encontrar en documentos institucionales una base empírica válida para el estudio de la realidad contemporánea.

La investigación documental se llevó a cabo a través de las siguientes etapas:

1. Búsqueda de información sobre el tema estudiado.

2. Clasificación de la información de acuerdo al lugar de su procedencia (América, Europa, entre otros). 
3. Análisis de la información por medio de su división en categorías homogéneas.

4. Elaboración de conclusiones a través del análisis categórico de los datos.

Los resultados del análisis de las diversas fuentes de información se presentan a continuación:

\section{Análisis de la literatura}

\section{Información sobre el tema}

El estudio del modelo de economía circular ha adquirido relevancia en todos los continentes desde el año 2000. En el continente asiático, el caso de China es el más emblemático, como comentan Prieto, Jaca y Ormazabal (2018) ya que, el $90 \%$ de los artículos sobre el tema fueron publicados después del año 2003 (153 documentos), cuando el gobierno comenzó a promover la implementación de la economía circular. La tendencia muestra que los temas de investigación relacionados con la economía circular son cada vez más importantes dentro de la comunidad científica de este país. Este resultado es evidencia de la clara influencia que China tiene en la investigación sobre el tema y su implementación.

En segundo lugar, con el mayor número de artículos publicados sobre la economía circular se encuentra la Unión Europea, mientras que en América Latina no existen estadísticas sobre las publicaciones con relación al tema. La mayor parte de las referencias halladas por ejemplo en México, se basan en artículos periodísticos y noticias que emiten información confusa con respecto a la implementación del modelo.

\section{Datos sobre la implementación del modelo}

Para comprender mejor la realidad con respecto al tema, se presentan los siguientes datos e indicadores:
1. En el año 2013, los beneficios económicos de la economía circular, asociados al ahorro en materias primas de las industrias, han sido cuantificados por la Comisión Europea en 600 000millones de euros (un $8 \%$ de la facturación anual de la Unión Europea). Los beneficios sociales como la creación de nuevos empleos han sido cuantificados por la Unión Europea en 580000 puestos de trabajo, de los cuales un 30 \% están asociados al pleno cumplimiento de la normativa comunitaria en materia de residuos (Ruíz et al, 2016).

2. En Europa, hay más de 4 millones de empresas que ya son parte de la economía circular, quienes generan facturas mayores a 147000 millones de euros. El deterioro del medioambiente se convierte en una oportunidad para las empresas, sobre todo para pymes y startups (El Economista, 2019).

3. En América Latina, la economía circular representa una oportunidad de negocio de 4.5 trillones de dólares. Se calcula que la región podrá crear 6 millones de empleos de calidad a partir de la economía circular (Plastich technology, 2019).

Como se observa en el análisis de datos, existen una gran cantidad de beneficios que las microempresas pueden adquirir al utilizar la economía circular, sin embargo, a la par existen una serie de retos que tienen que ser superados para poder gozar de estos beneficios. Por lo tanto, si bien la aplicación de estrategias circulares puede generar un beneficio a largo plazo es necesario contar con cimientos fuertes como microempresa ya que de otra forma se estaría perjudicando a los negocios en vez de beneficiarlos. El elevado costo inicial de la implementación del modelo, la falta de sensibilización de los consumidores con respecto al tema, la poca 
infraestructura y la poca capacidad de innovación son grandes obstáculos para que los microempresarios decidan implementar el modelo circular en sus negocios. El sistema económico y político de un país suelen ser determinante al momento de plantear estrategias de negocios. El microempresario prioriza su realidad inmediata sobre la situación medioambiental. En el juego de la supervivencia del negocio sobre el problema ambiental, el primero es, por lo general, el ganador.

Por lo tanto, para que la implementación de este modelo sea adecuada, es necesario que el gobierno y la sociedad puedan apoyar la ardua labor que un microempresario tiene que emprender para que los beneficios sean los deseados y que el país cuente con microempresas que produzcan beneficios económicos y cuiden el medioambiente. Los gobiernos deben de generar programas de capacitación y apoyos económicos directos para impulsar la implementación del modelo. La sociedad debe de exigir microempresas comprometidas con la labor ambiental y reconocer la labor de aquellas microempresas que han consolidado sus procesos de cambio de una estructura lineal a una circular. Por lo tanto, Preston (2012) menciona que existen una serie de pasos prácticos que los países y las empresas pueden adoptar para implementar estrategias de economía circular:

1. Mejorar las prácticas y el intercambio de conocimientos: intentando explicar a futuros inversores los beneficios de la aplicación de estrategias circulares.

2. Regulaciones inteligentes: que permitan y faciliten el intercambio de información entre el gobierno y la industria privada.

3. Estandarización: en los requerimientos necesarios para la compra de tecnología.

4. La siguiente generación de ideas: realizando un trabajo de triangulación en- tre los estudiantes, las universidades y la iniciativa privada.

\section{Casos de éxito en la implementación del modelo circular}

En el texto se han mencionado las principales estrategias para la implementación de la economía circular en la empresa, sin embargo no se han presentado ejemplos prácticos de la incorporación del modelo. Un ejemplo clásico es el planteado por Preston (2012), que comenta que la firma electrónica japonesa Kyocera fue una de las primeras en fabricar los cartuchos de tóner recargables. La empresa fabricaba cartuchos convencionales que eran desechados al final de su vida útil. En su lugar, creó cartuchos mucho más simples. Durante toda la vida del producto se generaron ahorros ya que el costo se reduce en un $50 \%$ (mientras que los residuos se reducen en un $90 \%$ ). Sin embargo, a pesar de sus esfuerzos en las últimas dos décadas, Kyocera admite que ha luchado para desplazar a los cartuchos convencionales como modelo de negocio. Lo anterior muestra como a pesar de que una empresa intentó implementar la economía circular por causa de los hábitos de consumo de los clientes, la estrategia no ha producido todos los beneficios que debió producir. Si bien, la firma es un negocio consolidado, la misma situación puede ocurrir con microempresas que intenten crear productos que modifiquen los hábitos de compra de los consumidores con el fin de ahorrar recursos. Los consumidores pueden no aceptar los cambios de buena manera a pesar de que se persiga un cambio deseable y positivo.

Otro ejemplo de la implementación de este modelo es el caso de un pequeño supermercado en Chiangmai, Tailandia Ilamado Rimping, en el que se utiliza la hoja de plátano (producto orgánico) como un envoltorio para sus frutas y verduras en lugar de utilizar bolsas de plástico. Lo anterior ha detonado 
la admiración de los clientes por su preocupación por el medioambiente y ha generado que el supermercado incremente su base de clientes leales. La innovación en este sentido, ha ocasionado un impacto positiva para el negocio, así como para el medioambiente. Sin embargo, los costos asociados a este cambio son elevados debido a que las tiras plásticas (contaminantes) son mucho más económicas en contraposición con la hoja de plátano natural.

Los casos anteriores permiten observar la forma en que la economía circular puede generar oportunidades y desafíos para cualquier tipo de negocio, ya sea grande o pequeño. Hasta el momento, en México, las principales incorporaciones de la economía circular a las microempresas en general, se han dado a través de procesos individuales $y$ aislados que surgen al interior de las mismas organizaciones. Los esfuerzos de asociaciones empresariales o del gobierno para que un mayor número de negocios adopten esta estrategia y se logren combatir los daños causados al medioambiente por el uso excesivo de los productos que provienen de la economía lineal son escasos.

\section{Conclusiones}

El modelo de economía lineal basado en el consumo masivo de materias primas para la producción debe de ser modificado. Esta situación no puede continuar de la misma forma ya que si el consumo de los recursos del planeta sigue creciendo de forma exponencial como hasta ahora, el planeta se quedará sin recursos en los próximos diez o quince años.

Se deben de buscar nuevas formas de producción y de consumo, por lo tanto, continuar con el actual modelo de economía lineal no es conveniente ni para el medioambiente ni para ningún ser vivo que habita en él. Los principales causantes de la contaminación en el mundo son las microempresas.
Si la mayor parte de las empresas son de carácter micro, es normal comentar que este tipo de negocio tiene la posibilidad de realizar un cambio para el planeta.

Por lo tanto, se deben de generar las condiciones necesarias para que las microempresas pasen de una economía lineal a una economía circular. Los beneficios de aplicar el modelo circular son muy variados, pero dicha implementación no es sencilla ya que implica una serie de barreras y desafíos que deben de ser superados. Desgraciadamente, en la realidad de los países subdesarrollados es más factible que las barreras no quieran ser rotas ni los desafíos superados debido a sus características: baja capacidad de producción, capital de trabajo insuficiente o la falta de fuentes de financiamiento. Por lo tanto, los principales desafíos para la implementación de la economía circular en las microempresas son: la baja producción, los altos costos de implementación, la falta de inversión y la visión a corto plazo de los empresarios, entre otros.

Las empresas latinoamericanas tienen que superar diversos retos (alta tasa de mortalidad, poca o nula capacidad de inversión, miedo al cambio y la administración de carácter tradicional) para poder incorporar la economía circular y disfrutar sus beneficios en el largo plazo. Para que las empresas de estos países puedan adherirse a este tipo de economía se requiere que existan inversores que conozcan los beneficios de las estrategias circulares, alentar el desarrollo de tecnología amigable con el ambiente y promover la participación de las universidades y sus alumnos en sectores importantes de la economía.

Derivado de lo anterior, es necesario que el sector gobierno adopte la postura de beneficiar al medioambiente sobre la producción lineal para que estas políticas se precisen como prioritarias y se promuevan ante los líderes de los organismos y cámaras empresariales de los diversos países 
para que sean implementadas en sus empresas. Se deben de promover y promulgar leyes que faciliten el acceso de las empresas a las ventajas de la economía circular. Pero de la misma forma como se espera que los organismos empresariales y el gobierno adopten posturas de apoyo al cambio de las microempresas lineales a microempresas circulares, también se espera que los consumidores y la población en general demanden productos y reconozcan la labor de las empresas circulares.

La labor de concientización depende de la creación de nuevas investigaciones con respecto a la materia para demostrar que es un tema prioritario, actual y pertinente en el campo de la investigación. Por este motivo, el presente artículo pretende convertirse en una evidencia y referencia de los cambios que deben producirse para que las microempresas den un paso hacia el futuro, dejen atrás los retos y los beneficios circulares permean en ellas. Como se menciona en el artículo, China y la Unión Europea son los líderes en le generación de información sobre la economía circular y América Latina, pero sobre todo México, se ha rezagado en producir información sobre este tema vital para la toma de decisiones. Por lo tanto, este artículo a pesar de ser un pequeño esbozo sobre el tema, espero que se convierta en el punto de partida para que otros investigadores de la región se interesen en generar más información sobre el mismo.

Como se observa en el texto, los desafíos y los beneficios de implementar la economía circular no varían demasiado sin importar que se trate de una empresa grande o de una empresa pequeña. Sin embargo, es muy importante que los negocios se encuentren preparados de forma adecuada para implementar los cambios que requieren las estrategias circulares, de lo contrario, lo mejor es no forzar la implementación y esperar a que se cuente con la estructura nece- saria al interior de la organización. Si la empresa no cuenta con la fortaleza financiera y administrativa necesaria, la utilización del modelo circular puede acelerar los procesos de desaparición de las mismas en lugar de convertirse en un incentivo para generar su crecimiento. El proceso de cambio debe de ser gradual y de acuerdo a las posibilidades de cada negocio.

\section{Referencias}

Comisión Europea para la Economía Circular. (2015). Capital natural: recursos físicos y biológicos, biodiversidad, suelos y ecosistemas. Recuperado de: http://ec.europa.eu/environment/circular-economy/index_en.htm)

Corbetta, P. (2007). Metodología y técnicas de investigación social. McGraw-Hill. Recuperado de: https://diversidadlocal.files.wordpress.com/2012/09/metodologc3ada-ytc3a9cnicas-de-investigacic3b3n-socialpiergiorgio-corbetta.pdf

Durán, P. (Julio, 2017). Microempresas, pymes y objetivos de desarrollo sostenible. Boletín ONU México. Recuperado de http://www. onu.org.mx/microempresas-pymes-y-objetivos-de-desarrollo-sostenible/

El Financiero. (abril, 2019). La economía circular y el reto de México en gestión de residuos. Recuperado de: http://www.aievac.org. mx/movil/nota/2546/la-economia-circular-y-el-reto-de-mexico-en-gestion-de-residuos

Espaliat, M. (2017). Economía circular y sostenibilidad. Nuevos enfoques para la creación de valor. Recuperado de: http://cewoliked. myddns.com/B07656NF33-EconomiaCircular-y-Sostenibilidad-Nuevos-enfoques-para-la-creacin-de-valor.pdf

Fundación Ellen MacArthur. (2017). Hacía una economía circular: motivos económicos para una transición acelerada. Recuperado de: https://www.ellenmacarthurfoundation.org/assets/downloads/publications/Executive_summary_SP.pdf

Garcés, C., Rivera, P., Suárez, I. y Leyva, D. (2019). Is it posible to change from a linear to a 
circular economy? An overview of opportunities and barriers for European small and medium sized enterprise companies. International Journal of Environmental Research and Public Health, 16(851), 3-15. DOl: 10.3390/ijerph16050851

García, M. y Ortega, A. (2018). Reparable, actualizable y reciclable. La economía circular, como nuevo modelo económico sustentable en México. Instituto de Investigaciones Económicas. Recuperado de: http:// ru.iiec.unam.mx/3961/

Kalmykova, Y., Sadagopan, M. y Rosado, L. (2018) Circular economy: From review of theories and practices to development of implementation tools. Resources, Conservation \& Recycling, 135(1), 190-201. Recuperado de: https://www.sciencedirect.com/ science/article/pii/S0921344917303701

Korhonen, J., Nuur, C., Feldmann, A. y Eshetu, S. (2018). Circular economy as an essentially contested concept. Journal of cleaner production, 175(1), 544-562. Recuperado de: https://www.sciencedirect.com/science/ article/pii/S0959652617330706

Lett, L. (2014). Las amenazas globales, el reciclaje de residuos y el concepto de economía circular. Revista Argentina de Microbiología, 46(1), 1-2. Recuperado de: http://www.redalyc.org/articulo.oa?id=213030865001

Ludeke, F., Gold, S. y Bocken, N. (2018). A review and typology of circular economy business model patterns. Journal of Industrial Ecology, 25(1), 36-61. DOl: 10.1111/ jiec.12763.

Organización de las Naciones Unidas (ONU). (1987). Informe de Brutland. Recuperado de: http://www.ecominga.uqam.ca/ PDF/BIBLIOGRAPHIE/GUIDE_LECTURE_1/ CMMAD-Informe-Comision-Brundtlandsobre-Medio-Ambiente-Desarrollo.pdf

Planing, P. (2015). Business model innovation in a Circular Economy: Reasons for nonacceptance of circular business models. Open journal of business model innovation, 1-11. Recuperado de: 273630392_Business_Model_Innovation_in_a_Circular_Economy_Reasons_for_Non
Plastich Technology México (abril, 2019). Economía circular una oportunidad de negocio. Recuperado de: https://www.pt-mexico. com/art\%C3\%ADculos/economia-circular-una-oportunidad-de-negocio-paramexico-y-al

Preston, F. (2012). A global redesign? Shaping the circular economy. Catham House, The Royal Institute of International Affairs. Recuperado de: https://www.chathamhouse. org/publications/papers/view/182376

Prieto, V., Jaca, C. y Ormazabal, M. (2017). Economía circular: relación con la evolución del concepto de sostenibilidad y estrategias para su implementación. Memorias en Investigaciones en Ingeniería, 15(1), 85-95. Recuperado de: https://dialnet.unirioja. es/servlet/articulo?codigo $=6296083$

Prieto, V., Jaca, C. y Ormazabal, M. (2018). Towards a consensus on the circular economy. Journal of cleaner production, 179(1), 605-615. Recuperado de: https:// www.sciencedirect.com/science/article/ pii/S0959652617332146

Programa Nacional de Financiamiento al Microempresario en México (PRONAFIM). (2002). El rol de la microempresa en México. Recuperado de: https://www.microfinancegateway.org/sites/default/files/ mfg-es-documento-el-rol-de-la-microempresa-en-mexico-2002.pdf

Quecedo, R. y Castaño, C. (2002). Introducción a la metodología de investigación cualitativa. Revista de psico didáctica, 1(14), 5-39. Recuperado de: https://www.redalyc.org/ pdf/175/17501402.pdf

Ruíz, M., Fabrellas, B., Dávila, S., Santervas, G., Cabrera, A., Gonzalo, G., Tapia, C., \& Callaba, A. (2016). La economía circular. Revista ambientia, 125(1), 3-11. Recuperado de: https://www.mapa.gob.es/ministerio/ pags/biblioteca/revistas/pdf_AM/PDF_AM_ Ambienta_2016_117_completa.pdf

Sánchez, G. (2007). Perspectivas de las micro y pequeñas empresas como factores del desarrollo económico de México. Recuperado de: http://www.economia.unam.mx/ profesor/barajas/perspec.pdf 
Secretaría de Economía de México (SE). (2010). Microempresas. Recuperado de: http:// www.2006-2012.economia.gob.mx/mexico-emprende/empresas/microempresario Stake, R. (1998). Investigación con estudio de casos. Madrid: editorial Morata. Recuperado de: https://www.uv.mx/rmipe/files/2017/02/Investigacion-con-estudiosde-caso.pdf
Veolia México. (2018). La economía circular: un modelo que permitirá el crecimiento de las empresas. Revista de la industria energética. Recuperado de: https://petroquimex.com/la-economia-circular-unmodelo-que-permitira-el-crecimiento-delas-empresas/ 